\title{
3D Display and Analysis of Strain Fields at Heterointerfaces
}

\author{
G. Möbus*, A. Trampert** \\ *Sheffield University, Dept of Engineering Materials, Sheffield, S1 3JD, UK \\ ** Paul-Drude-Institut, Berlin, Germany
}

HREM images of lattice mismatched heterointerfaces can be analysed by a variety of image processing techniques, including moire-patterns, peak-position mapping, 1D-Fourier spectra, geometrical phase mapping, or iterative matching with HREM-simulations [1-6]. For samples with misfit dislocation distance $L$ smaller than specimen thickness, the problem becomes complex and three-dimensional due to projection effects, and would require a 3D structure model with thousands of unknowns [7-8]. Disregarding these complications, we discuss in this work display techniques for strain fields at heterointerfaces, which do not operate on atomic level, but allow the derivation (at best) of a set of simple parameters ranging on interval $[0,1]$ to classify various heterointerfaces in comparison. The example of FIG 1 is a (100) InAs/GaAs semiconductor heterointerface [9].

The parameters searched for, which could be seen as spanning a 5-dim. configuration space are:

(1) actual misfit / long range strain field, defined by the fraction $F$ of actual/theoretical misfit.

(2) Amplitude $A$ of the fundamental displacement wave within $D=A d / 2 \cos (2 \pi x / L)$.

(3) Ratio $B$ between $A_{1}$ on "rigid" and $A_{2}$ on "soft" side for elastically dissimilar crystals.

(4) Ratio $\mathrm{C}$ between $A_{\mathrm{y}}$ and $A_{\mathrm{x}}$ for displacement waves with components in and out of interface.

(5) Localisation: Ratio of $2^{\text {nd }}$ to $1^{\text {st }}$ harmonic of displacement wave (or ratio $W$ of $F W H M$ to $L$ ). More details of this work will appear in [10]. Motivation for such Fourier-expansion analysis can be found in recent continuum modelling approaches [11,12]. We display geometrical phase maps $[4,5]$ in surface representation, with the height of the surface $[-\pi ;+\pi]$ indicating a lateral shift of the lattice with respect to the reference area in selected Bragg-peak direction. The reference lattice becomes a (more or less) flat plane, the opposing mismatched lattice a saw-tooth plane with every $2 \pi$ - phase-jump being an extra plane inserted. The interface connects singularities ( possible dislocations). The extent of strain into the reference crystal indicates deviation from incoherence towards partial coherence. Profile plots can be extracted for measurements of A,B,C,W. HREM delocalisation prevents measurement at the interface and requires at least one point-spread distance. High-voltage, Cs-correction, or focal series reconstruction will improve accuracy. [13]

[1] C.J.D. Hetherington, U. Dahmen, Scann. Microsc. Suppl. 6 (1992) 405.

[2] R. Bierwolf et al. Ultramicroscopy 49 (1993) 273.

[3] G. Möbus, E. Schumann, G. Dehm, M. Rühle, phys. stat. sol. (a) 150 (1995) 77.

[4] M.J. Hÿtch, E. Snoeck, R. Kilaas, Ultramicroscopy 74 (1998) 131.

[5] E. Snoeck et al., Thin solid films 319 (1998) 157.

[6] G. Möbus, T. Wagner, J. Microsc. 194 (1999) 124.

[7] H.B. Groen et al. Phil. Mag. A 79 (1999) 2083.

[8] A. Levay et al., Acta Mater. 47 (1999) 4143.

[9] A. Trampert, K.H. Ploog, E. Tournie, Appl. Phys. Lett. 73 (1998) 1074.

[10] G. Möbus et al., Z.f Metallkunde, submitted (2003).

[11] A.E. Romanov, T.Wagner, M. Rühle, Scripta Mater. 38 (1998) 869.

[12] F. Ernst, R. Raj, M. Rühle, Z. Metallkd. 90 (1999) 961.

[13] This work was funded by EPSRC (\# GR-M81786 and AF-98-1532). 


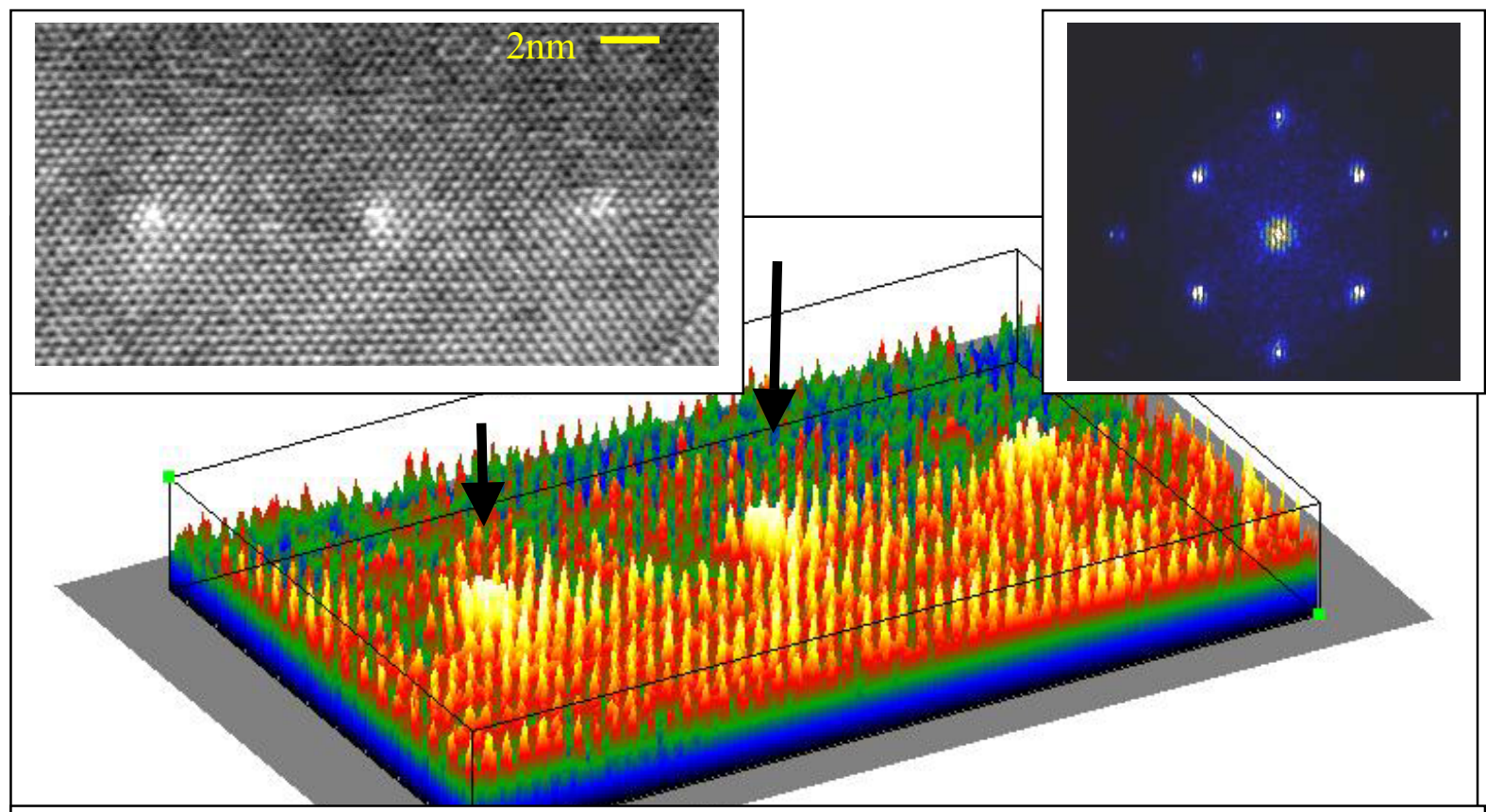

FIG. 1. InAs[110], bottom // GaAs[110], top;(100) interface. Top left: HREM (JEM4000EX; From [9]). Top right: Diffractogram with split $\{111\}$-peaks selected for processing into FIG 2. Large image: 3D-pseudocoloured HREM image surface display with extra half planes arrowed.
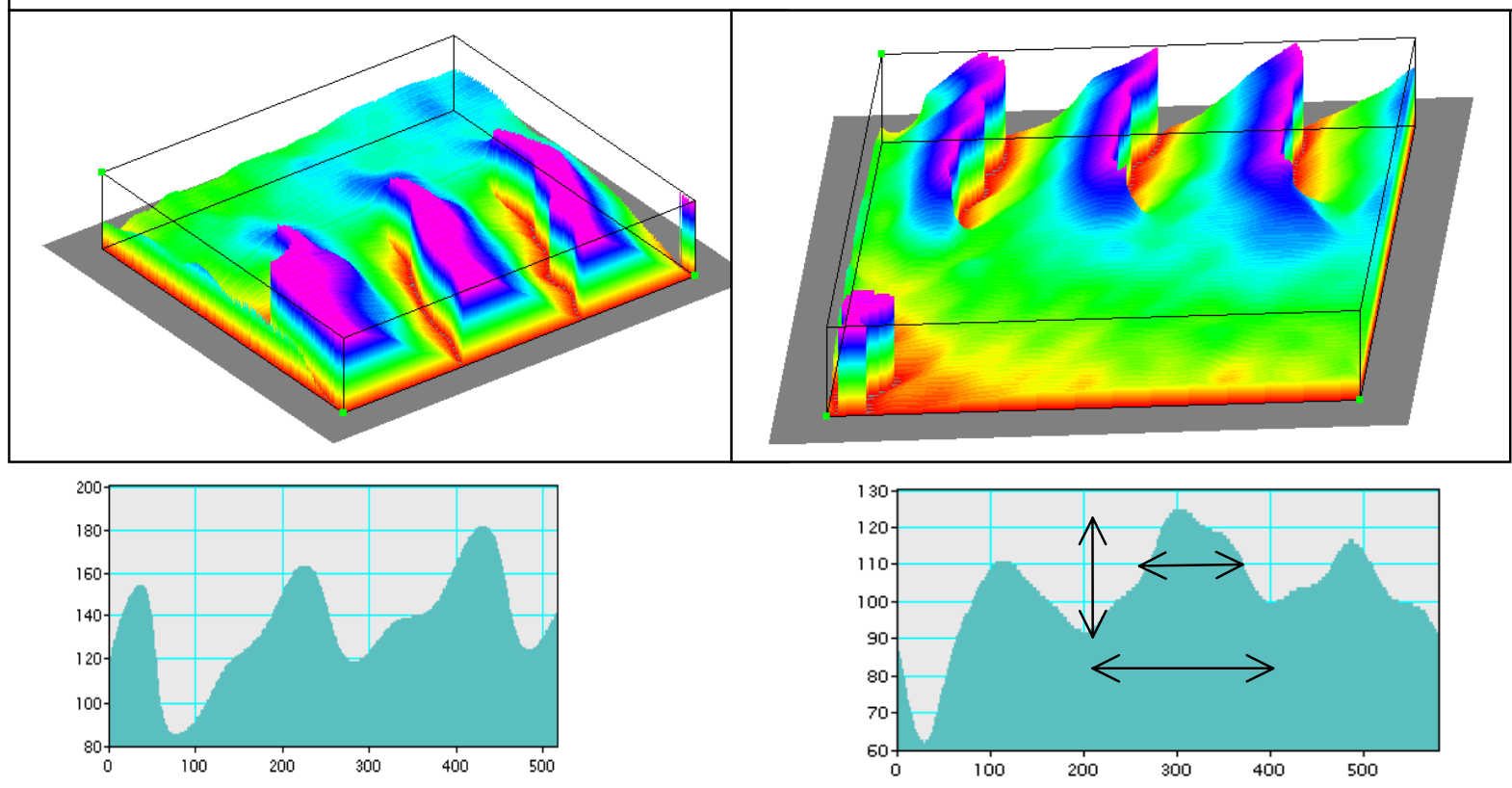

FIG. 2. (a) Geometric phase map using InAs $\{111\}$ reflection: upper-far half is InAs reference (green, strain regions are blue), bottom-near half is sawtooth-phase of mismatched GaAs. (b) using GaAs $\{111\}$ : upper-far half is mismatched InAs-sawtooth surface. Bottom-near plane is GaAs reference plane (green) with strain fields (blue). Bottom-left corner is stacking fault artefact. Profiles below show displacement fields at one point spread distance from the interface on the reference lattice side. Double arrows indicate values to be measured for determination of the parameters $A, L, W$. The long range of extend of the strain fields into the reference lattice (blue colour in $a, b)$ is typical for covalent heterointerfaces, as opposed to metal/ceramic interfaces. 